\title{
Ring-Doped Tm Fibres for High-Efficiency Cladding-Pumped 1907 nm Lasers
}

\author{
Matthew J. Barber ${ }^{1 *}$, Peter C. Shardlow ${ }^{1}$, Pranabesh Barua ${ }^{1}$, Jayanta K. Sahu ${ }^{1}$ and \\ W. Andrew Clarkson ${ }^{1}$ \\ ${ }^{1}$ Optoelectronics Research Centre, University of Southampton, Southampton, SO17 1BJ, United Kingdom \\ *m.j.barber@soton.ac.uk
}

\begin{abstract}
A cladding-pumped Tm fibre is designed and fabricated with a ring-structured fibre core geometry, optimised for power-scalable, single-mode $1907 \mathrm{~nm}$ operation with $67.0 \%$ slope efficiency and demonstrated in an all-fibre laser oscillator configuration. 2019 The Author(s)
\end{abstract}

OCIS codes: $140.3070,140.3510$

Laser sources emitting at $1907 \mathrm{~nm}$ are ideally suited to pumping Ho:YAG, whose laser output can be frequency-converted in an optical parametric oscillator (OPO) to access the mid-infrared regime. High-power mid-infrared emission offers potential applications in highly sensitive spectroscopy, transparent plastics processing and all-optical defensive countermeasure systems [1]. At present, the achievable output powers from this route to the mid-infrared are limited by the difficulty in power scaling single-mode $1907 \mathrm{~nm}$ sources. Here, we report on nesting a high concentration ring of Tm doped core material within a larger undoped fibre core. This approach is shown to provide higher efficiency and higher power scalability than is possible with classical uniformly doped Tm fibre core designs.
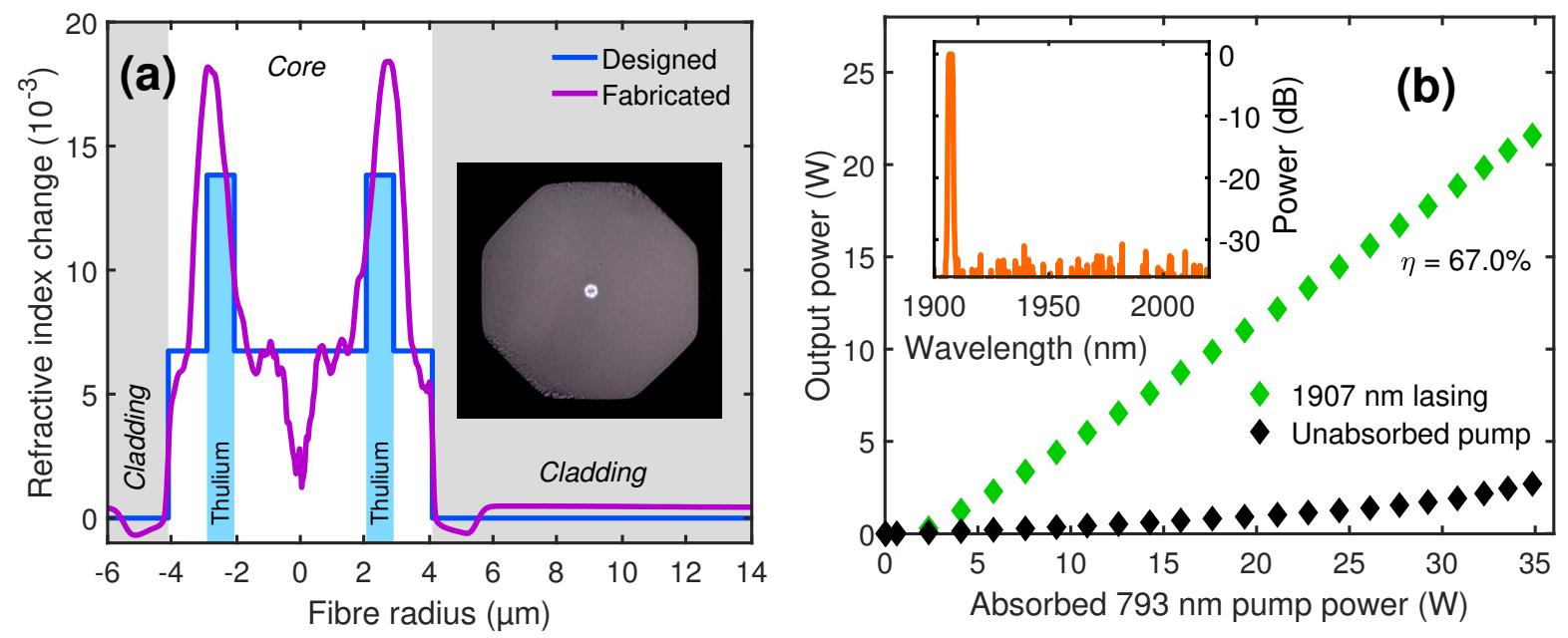

Fig. 1. (a) Refractive index profile of the ring-structured-core fibre. Inset: end-face image of the ring-structured-core fibre. (b) Output power curve of the Tm-doped fibre with the corresponding slope efficiency with respect to absorbed power, $\eta$. Inset: $1907 \mathrm{~nm}$ emission spectrum at $21.6 \mathrm{~W}$ output.

To operate Tm-doped fibre lasers at short wavelengths, high population inversions are required to counteract the strong reabsorption losses that arise due to the three level nature of the transition. However, parasitic lasing and amplified spontaneous emission (ASE) at longer wavelengths also extract gain from the excited Tm core. This limits the maximum inversion levels and prevents short wavelength lasing. This can be overcome by minimising the total number of ions within the core path of the fibre laser, such as by a reduction in the fibre length. Therefore, the gain for longer wavelength parasitics is reduced, but at the expense of a lower pump absorption efficiency. For $1907 \mathrm{~nm}$ operation, both core pumping at $1550 \mathrm{~nm}$ and close cladding-to-core area ratio pumping at 79x $\mathrm{nm}$, have been reported. However, Tm-doped fibres pumped at $1550 \mathrm{~nm}$ are limited to low power emission due to the high-brightness requirements of the pump source. To achieve high population inversions through 
cladding-pumping, a small cladding-to-core area ratio is used. As a consequence, this leads to shorter device lengths due to the high pump absorption. This in turn leads to high thermal loading, limiting the power scalability. A reduction in Tm doping concentrations (to $<2 \mathrm{wt} \%$ ) can allow the fibre device length to be increased, however this sacrifices the two-for-one cross-relaxation process that can be exploited in highly doped Tm fibres. At present, single-mode cladding-pumped Tm-doped fibre lasers reported at $1907 \mathrm{~nm}$ have achieved slope efficiencies of up to $53 \%$ [2,3]. Here, we explore a nested Tm-doped ring-structured core dopant profile, as shown in Figure 1a. The core is comprised of highly doped Tm layers as well as undoped layers. This replaces the uniform low concentration doping found in the current generation of $1907 \mathrm{~nm}$ Tm-doped fibre lasers. From the high concentration ring-structured Tm doping, a strong two-for-one cross-relaxation process is maintained whilst the undoped core material reduces the pump absorption per unit length. This allows longer device lengths to be used, improving the thermal management of the fibre. In addition, the confinement of Tm ions to a ring geometry around the centre of the core ensures the guided fundamental mode has a lower overlap factor with the doped region. This suppresses the signal reabsorption losses and permits easier access to the short wavelength regime.

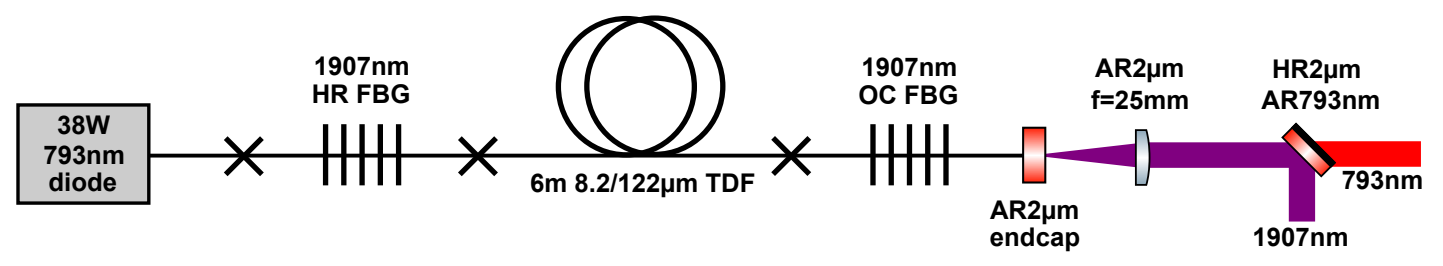

Fig. 2. Tm-doped fibre (TDF) cavity wavelength-locked for $1907 \mathrm{~nm}$ operation.

The fibre was fabricated in-house via solution-doped MCVD (modified chemical vapour deposition) with a mix of Ge-doped and solution-doped Tm \& Al layers, as shown in Figure 1a. The ring-structured-core fibre was drawn to a $8.2 \mu \mathrm{m}$ diameter core for robust single-mode operation at $1907 \mathrm{~nm}$, matching the mode field diameter to passive $10 \mu \mathrm{m} / 0.15 \mathrm{NA}$ fibre. The fibre cladding was octagonally-shaped for efficient pump mode scrambling and drawn to area match the passive fibre, with a $122 \mu \mathrm{m}$ flat-to-flat distance and a $132 \mu \mathrm{m}$ corner-to-corner distance. A single 6 metre device length of ring-structured-core fibre was cladding-pumped at $793 \mathrm{~nm}$ and locked to a $1907 \mathrm{~nm}$ high reflectivity (HR) and output coupler (OC) fibre Bragg grating (FBG) pair (Figure 2). The output port of the fibre chain is bonded to a $2 \mu \mathrm{m}$ anti-reflection coated end cap. This fibre laser system demonstrated $67.0 \%$ slope efficiency with respect to absorbed pump power and $62.1 \%$ slope efficiency with respect to launched pump power. The fibre laser produced $21.6 \mathrm{~W}$ of $1907 \mathrm{~nm}$ output power from $37.5 \mathrm{~W}$ of launched pump light (Figure 1b). The fibre had $11.4 \mathrm{~dB}$ pump absorption $(1.90 \mathrm{~dB} / \mathrm{m})$ and $2.7 \mathrm{~W}$ of unabsorbed pump at full power. Beam quality factor $\left(\mathrm{M}^{2}\right)$ measurements of $0.98 \pm 0.05$ were observed in both axes, consistent with single-mode operation. Figure $1 \mathrm{~b}$ (inset) confirms parasitic-free and ASE-free laser operation at $1907 \mathrm{~nm}$.

To the best of our knowledge, this is the most efficient demonstration of a $1907 \mathrm{~nm}$ Tm-doped cladding-pumped silica fibre laser, with efficiencies up to $67 \%$ with respect to absorbed pump power. In comparison to reported fibre lasers with uniform doping profiles, the ring-structured core has produced a three-fold increase in fibre device length, a $25 \%$ increase in slope efficiency and a correlated reduction of the quantum defect heating. Collectively, this demonstrates a 4.5 times improvement in the power scalability of short wavelength cladding-pumped Tm fibre lasers. The use of localised, high-concentration Tm doping in a ring-structured-core geometry has produced slope efficiencies exceeding those available from classical uniformly-doped fibres at this operating wavelength, offering the possibility of high efficiency multi-100 W-class $1907 \mathrm{~nm}$ lasers with single-mode emission from a single oscillator.

\section{References}

1. X. Mu et al. High-efficiency high-power 2.097- $\mu \mathrm{m}$ Ho:YAG laser pumped mid-infrared ZGP optical parametric oscillation. Laser Technology for Defense and Security VI, 7686, 76860T (2010).

2. G. Frith et al. Highly efficient $70 \mathrm{~W}$ all-fibre Tm-doped laser system operating at $1908 \mathrm{~nm}$. OECC/ACOFT 2008 - Joint Conference of the Opto-Electronics and Communications Conference and the Australian Conference on Optical Fibre Technology, pp. 1-2 (2008).

3. B. R. Johnson et al. Comparison of high power large mode area and single mode $1908 \mathrm{~nm}$ Tm-doped fiber lasers. Proc. SPIE 9728, Fiber Lasers XIII: Technology, Systems, and Applications, 972810 (2016). 